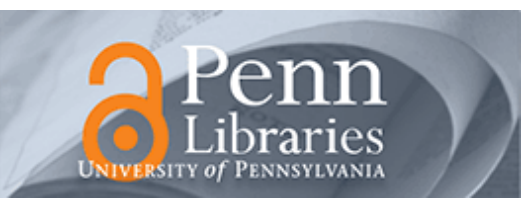

University of Pennsylvania ScholarlyCommons

3-13-2012

\title{
Thermomechanical Stability of Ultrananocrystalline Diamond
}

\author{
Vivekananda P. Adiga \\ University of Pennsylvania, adiga@seas.upenn.edu \\ Sampath Suresh \\ Innovative Micro Technology \\ Arindom Datta \\ Innovative Micro Technology \\ John A. Carlisle \\ Advanced Diamond Technology \\ Robert W. Carpick \\ University of Pennsylvania, carpick@seas.upenn.edu
}

Follow this and additional works at: https://repository.upenn.edu/mse_papers

Part of the Condensed Matter Physics Commons, and the Other Materials Science and Engineering Commons

\section{Recommended Citation}

Adiga, V. P., Suresh, S., Datta, A., Carlisle, J. A., \& Carpick, R. W. (2012). Thermomechanical Stability of Ultrananocrystalline Diamond. Retrieved from https://repository.upenn.edu/mse_papers/216

Adiga, V. P., Suresh, S., Datta A., Carlisle, J. A., \& Carpick, R. W. (2012). Thermomechanical stability of ultrananocrystalline diamond. Journal of Applied Physics, 111(5), 054913. doi: 10.1063/1.3693308 (C) 2102 American Institute of Physics. This article may be downloaded for personal use only. Any other use requires prior permission of the author and the American Institute of Physics. The following article appeared in J. Appl. Phys. 111, 054913 (2012) and may be found at http://link.aip.org/link/?jap/111/054913

This paper is posted at ScholarlyCommons. https://repository.upenn.edu/mse_papers/216

For more information, please contact repository@pobox.upenn.edu. 


\title{
Thermomechanical Stability of Ultrananocrystalline Diamond
}

\author{
Abstract \\ We have measured mechanical stiffness and dissipation in ultrananocrystalline diamond (UNCD) from 63 \\ $\mathrm{K}$ to $450 \mathrm{~K}$ using microcantilever resonators in a custom ultrahigh vacuum (UHV) atomic force \\ microscope. UNCD exhibits a temperature coefficient of modulus that is found to be extremely low: -26 \\ $\mathrm{ppm} / \mathrm{K}$, which is close to the previously measured value of $-24 \mathrm{ppm} / \mathrm{K}$ for single crystal diamond. The \\ magnitude and the temperature dependence of dissipation are consistent with the behavior of disordered \\ systems. The results indicate that defects, most likely at the grain boundaries, create the dominant \\ contribution to mechanical dissipation. These measurements of modulus and dissipation versus \\ temperature in this temperature range in UNCD establish the nanostructure's effect on the \\ thermomechanical stability and suggest routes for tailoring these properties.

\section{Keywords} \\ elastic modulus, dissipation, Q, resonators, ultrananocrystalline diamond \\ Disciplines \\ Condensed Matter Physics | Other Materials Science and Engineering

\section{Comments} \\ Adiga, V. P., Suresh, S., Datta A., Carlisle, J. A., \& Carpick, R. W. (2012). Thermomechanical stability of \\ ultrananocrystalline diamond. Journal of Applied Physics, 111(5), 054913. doi: 10.1063/1.3693308 \\ (C) 2102 American Institute of Physics. This article may be downloaded for personal use only. Any other \\ use requires prior permission of the author and the American Institute of Physics. The following article \\ appeared in J. Appl. Phys. 111, 054913 (2012) and may be found at http://link.aip.org/link/?jap/111/ \\ 054913
}




\title{
Thermomechanical stability of ultrananocrystalline diamond
}

\author{
Vivekananda P. Adiga, ${ }^{1, a)}$ Sampath Suresh, ${ }^{2}$ Arindom Datta, ${ }^{2, b)}$ John A. Carlisle, ${ }^{3}$ and \\ Robert W. Carpick ${ }^{4, c)}$ \\ ${ }^{1}$ Department of Materials Science and Engineering, University of Pennsylvania, Philadelphia, Pennsylvania \\ 19104, USA \\ ${ }^{2}$ Innovative Micro Technology, Santa Barbara, California, 93117, USA \\ ${ }^{3}$ Advanced Diamond Technologies, Romeoville, Illinois 60446, USA \\ ${ }^{4}$ Department of Mechanical Engineering and Applied Mechanics, University of Pennsylvania, Philadelphia, \\ Pennsylvania, 19104, USA
}

(Received 11 October 2011; accepted 11 February 2012; published online 13 March 2012)

\begin{abstract}
We have measured mechanical stiffness and dissipation in ultrananocrystalline diamond (UNCD) from $63 \mathrm{~K}$ to $450 \mathrm{~K}$ using microcantilever resonators in a custom ultrahigh vacuum (UHV) atomic force microscope. UNCD exhibits a temperature coefficient of modulus that is found to be extremely low: $-26 \mathrm{ppm} / \mathrm{K}$, which is close to the previously measured value of $-24 \mathrm{ppm} / \mathrm{K}$ for single crystal diamond. The magnitude and the temperature dependence of dissipation are consistent with the behavior of disordered systems. The results indicate that defects, most likely at the grain boundaries, create the dominant contribution to mechanical dissipation. These measurements of modulus and dissipation versus temperature in this temperature range in UNCD establish the nanostructure's effect on the thermomechanical stability and suggest routes for tailoring these properties. C 2012 American Institute of Physics. [http://dx.doi.org/10.1063/1.3693308]
\end{abstract}

\section{INTRODUCTION}

Nanocrystalline materials exhibit mechanical properties markedly distinct from their bulk analogs due to the nature of the bonds at the grain boundaries. ${ }^{1,2}$ The effect of nanocrystallinity is particularly interesting to study in diamond, because the tetrahedral $\mathrm{sp}^{3}$-hybridized carbon bonds of crystalline diamond provide the highest atomic density of all carbon allotropes as well as superior physical properties including the high Young's modulus, hardness, melting temperature, and acoustic velocity, and among the highest thermal conductivities and lowest thermal expansion coefficients. Single crystal diamond is one of the most thermomechanically stable materials known, but is difficult to integrate with complementary metal oxide semiconductor (CMOS) devices which require thin film geometries. However, uniform, thin polycrystalline diamond films known as ultrananocrystalline diamond (UNCD) can be grown using processes and temperatures compatible with such devices. UNCD films can retain the high elastic modulus, ${ }^{3}$ high acoustic velocity, ${ }^{3}$ and chemically stable surface of single crystal diamond. ${ }^{4}$ Consequently, UNCD is promising for high frequency microelectromechanical systems (MEMS)/ nanoelectromechanical systems (NEMS) devices ${ }^{3}$ such as filters, oscillators, and resonant mass sensors and biosensors. ${ }^{5}$ All of these require high temperature stability of the mechanical properties: thermal fluctuations in Young's modulus will affect the resonant frequency, resulting in poor performance

\footnotetext{
a) Author to whom correspondence should be addressed. Electronic mail: vpa8@cornell.edu. Present address, 212 Clark Hall, Applied and Engineering Physics, Cornell University, Ithaca, NY, 14850, USA.

${ }^{b)}$ Present address, Veeco Instruments Inc., 1 Terminal Drive, Plainview, NY 11803, USA.

c) Author to whom correspondence should be addressed. Electronic mail: carpick@seas.upenn.edu.
}

of high frequency filters and oscillators, for example, overwhelming the frequency shift due to adsorbed mass in sensors. How the UNCD nanostructure causes its thermomechanical behavior to deviate from single crystal diamond is unknown. In fact, experimental investigations of the temperature dependence of elastic properties are relatively scarce even for single crystal diamond. ${ }^{6,7}$ This is primarily a result of the fact that changes in the elastic modulus of single crystal diamond is less than $0.1 \%$ from room temperature to $0 \mathrm{~K}$, and is therefore challenging to measure. ${ }^{7}$ Furthermore, the uncertain grain boundary structure of UNCD renders these properties difficult to predict or simulate. This motivates experimental measurement of these properties as a function of temperature. Low temperature measurements are also helpful in extracting several material parameters such as the Grüneisen constant ${ }^{7}$ and the temperature dependence of the specific heat. ${ }^{8,9}$ As well, measurements of mechanical dissipation at low temperatures are necessary to identify physical mechanisms responsible for the quality factor $(Q)$ of resonators observed at room temperature, ${ }^{10}$ which is also important for applications in filters, oscillators, and mass sensors.

\section{FABRICATION OF DEVICES AND EXPERIMENTAL METHODS}

Recently, we reported the room temperature Young's modulus $(E)$, Poisson's ratio $(\nu)$, and $Q$ of microfabricated overhanging ledges and fixed-free beams fabricated from UNCD films grown at $680{ }^{\circ} \mathrm{C}^{10}$ The growth and characterization of the UNCD films on silicon substrates and the subsequent fabrication of the cantilever devices and the measurement methodology have been presented in detail elsewhere. ${ }^{10}$ Briefly, cantilevers were released using deep reactive ion etch (DRIE), followed by an isotropic $\mathrm{SF}_{6}$ etch. 
This allowed a much larger gap $(\sim 150 \mu \mathrm{m})$ between the levers and the underlying silicon to be produced without adding substantially to the undercut at the cantilever base. Larger gaps are necessary to enable atomic force microscope (AFM) measurements, where one must distinguish between the AFM laser light signal reflected from the less reflective UNCD cantilevers rather than silicon substrate. Overhangs were further reduced by focused ion beam (FIB) milling at the cantilever base. Large overhangs $(>55 \mu \mathrm{m})$ result in stress relaxation resulting in undulations whose amplitude and period depend on residual stress, which changes with temperature due to thermal mismatch resulting in changes in the amplitude and period of the undulations. Reducing the undercut of the UNCD film at the cantilever base is necessary to eliminate the influence of residual film stresses on the resonant frequency of the cantilever while it undergoes cooling and heating. ${ }^{11}$

Resonant excitation and ring-down measurements of the cantilevers were conducted on a custom-built stage in a RHK 750 UHV (pressure $<5 \times 10^{-10}$ Torr) AFM to determine the Young's modulus and $Q$ values of the UNCD cantilevers. $Q$ values are also determined by measuring the full width at half maximum (FWHM) at the fundamental flexural resonant frequency through curve fitting a Lorentzian to amplitude versus drive frequency data obtained from a phase-lock loop signal detection system (PLL Pro, RHK Technology Inc.). Details of the stage design are discussed elsewhere. ${ }^{10} \mathrm{UHV}$ conditions were necessary to prevent condensation of molecular contaminants on the resonators, which is expected when the temperature is decreased. In addition, viscous damping due to surrounding gases, which would dominate the response of these cantilevers at ambient pressure, is eliminated in UHV. Measurements were conducted from $450 \mathrm{~K}$ to $63 \mathrm{~K}$, and then repeated from $63 \mathrm{~K}$ to $450 \mathrm{~K}$ to detect any hysteresis (none was observed). The heating and cooling rates were approximately $1 \mathrm{~K} / \mathrm{min}$ and the temperature was stabilized before each measurement.

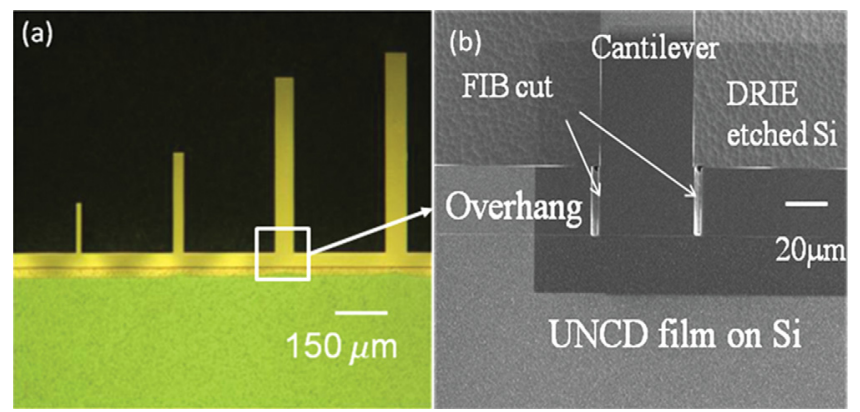

FIG. 1. (Color online) (a) Bright-field optical microscope image of a typical set of released UNCD cantilever resonator beams with an overhang. As a result of optical interference, the free standing UNCD and UNCD on silicon substrate show a color contrast (see the online version). (b) SEM image of a single cantilever beam. FIB milling was conducted at the cantilever base to reduce or eliminate any overhang, as indicated. Portions of the UNCD appear with darker contrast simply due to charging of a previously SEMimaged region.

\section{RESULTS AND DISCUSSION}

Figure 1 shows a typical cantilever, one of many we have fabricated and characterized with primary flexural resonance frequencies in $\mathrm{kHz}$ regime. The resonant frequency $f_{n}$ for the $n^{\text {th }}$ mode of an undamped, freely vibrating prismatic (constant cross section) linear-elastic cantilever with a rectangular cross section is given by,

$$
f_{n}=\frac{\beta_{n}^{2}}{2 \pi} \frac{t}{L^{2}} \sqrt{\frac{E}{12 \rho}},
$$

where $L, t$, and $\rho$ are the length, thickness, and mass density of the beam, respectively; $E$ is Young's modulus; and $\beta_{n}=\sim 1.875$ for $n=1$. From room temperature measurements of $f$, we found that $E=790 \pm 30 \mathrm{GPa}, \sim 20 \%$ lower than the theoretically predicted value for randomly oriented polycrystalline diamond. ${ }^{10}$ This difference is attributable to the high density of grain boundaries in UNCD. ${ }^{2,3,10}$ Structural and spectroscopic studies of other UNCD films (with somewhat different growth conditions) estimate that $\sim 10 \%$ of the $\mathrm{C}$ atoms reside at grain boundaries. ${ }^{12}$ Their contribution to temperature-dependent elastic properties is unknown.

We measured shifts in the resonant frequency (and sometimes, the $1^{\text {st }}$ harmonic) of the cantilevers versus temperature in UHV to extract the minute changes expected in the modulus. Figure 2(a) shows a typical measurement, from $63 \mathrm{~K}$ to $450 \mathrm{~K}$. The temperature coefficient of frequency (TCF) is commonly used to compare the temperaturedependent resonant frequency of structures made from materials without reference to their specific structural

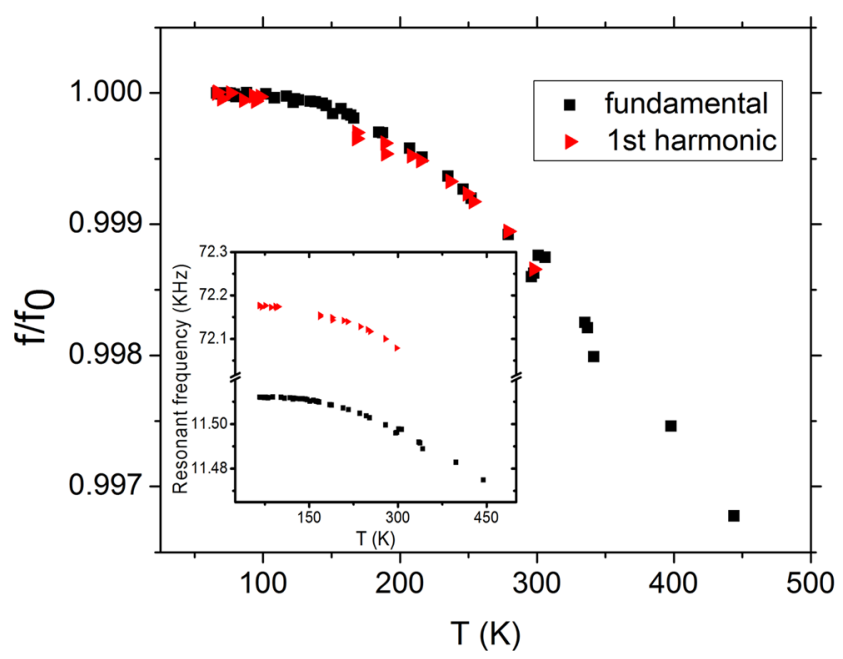

FIG. 2. (Color online) Representative data for the frequency shift relative to the low temperature limiting value and the absolute resonant frequency (inset) as a function of temperature for the fundamental and the first harmonic for a typical UNCD cantilever (in this case, $460 \mu \mathrm{m}$ long with no overhang). Measurements were obtained on three different levers with varying lengths ( $350 \mu \mathrm{m}$ to $460 \mu \mathrm{m}$ ) and overhangs (no overhang to $30 \mu \mathrm{m}$ overhang). This particular cantilever has had more data points and a broader temperature range over which the data has been collected, and hence these data are used for calculations, but results were consistent between cantilevers. These plots include data points taken during both heating and cooling cycles (individual cantilevers have gone through at least three cycles), and are reproducible. Heating and cooling rates were approximately $1 \mathrm{~K} / \mathrm{min}$. Higher order modes of the cantilevers also show a similar relative temperature dependence in terms of frequency and, hence, modulus. 
geometry. It is defined as $T C F=\frac{\Delta f}{f_{0} \Delta T}$, where $f_{0}$ is the resonant frequency at $300 \mathrm{~K}$, and $\Delta f$ is the shift in the resonant frequency over the measured temperature range, which is from $260 \mathrm{~K}$ to $320 \mathrm{~K}(\Delta T=60 \mathrm{~K})$. The TCF of UNCD measured $(-13.5 \mathrm{ppm} / \mathrm{K})$ is extremely low, among the lowest values observed among common MEMS/NEMS materials (e.g., single crystal silicon is $-51 \mathrm{ppm} / \mathrm{K}) .{ }^{13}$ Shifts in resonant frequency are correlated to changes in $E$ through Eq. (1). Taking the derivative of Eq. (1) and rearranging the terms yields, ${ }^{11}$

$$
\frac{1}{E} \frac{d E}{d T}=2 \frac{1}{f} \frac{d f}{d T}-\alpha
$$

where $\alpha$ is the coefficient of thermal expansion. The TCF of materials is roughly equal to half of the temperature coefficient of elastic modulus (TCE). Dimensional changes due to thermal contraction/expansion and thermal changes in density contribute to the TCE by an amount of the order of the coefficient of thermal expansion $(\sim 1 \mathrm{ppm} / \mathrm{K})$. Figure 3(a) shows the temperature-dependent shift in the modulus of UNCD relative to the low temperature limit determined from the data in Fig. 2, compared with the only available equivalent measurements for single crystal diamond (for proper comparison, the single crystal diamond modulus has been averaged over all directions by Voigt-Reuss-Hill approximation). We used previously published values of the thermal expansion of single crystal diamond as a function of temperature $^{14}$ to extract the relative temperature dependence of UNCD. ${ }^{11}$ At room temperature, the thermal stability of $E$ for $\mathrm{UNCD}$ is found to be $-26 \mathrm{ppm} / \mathrm{K}$. This is remarkably comparable with single crystal diamond $(-24 \mathrm{ppm} / \mathrm{K})$ given the significant fraction of grain boundary atoms in UNCD. For comparison, elevated temperature $(>300 \mathrm{~K})$ measurements of resonant frequency shifts in resonators made from tetrahedral amorphous carbon (ta-C), ${ }^{15}$ for which $\sim 80 \%$ of the car-

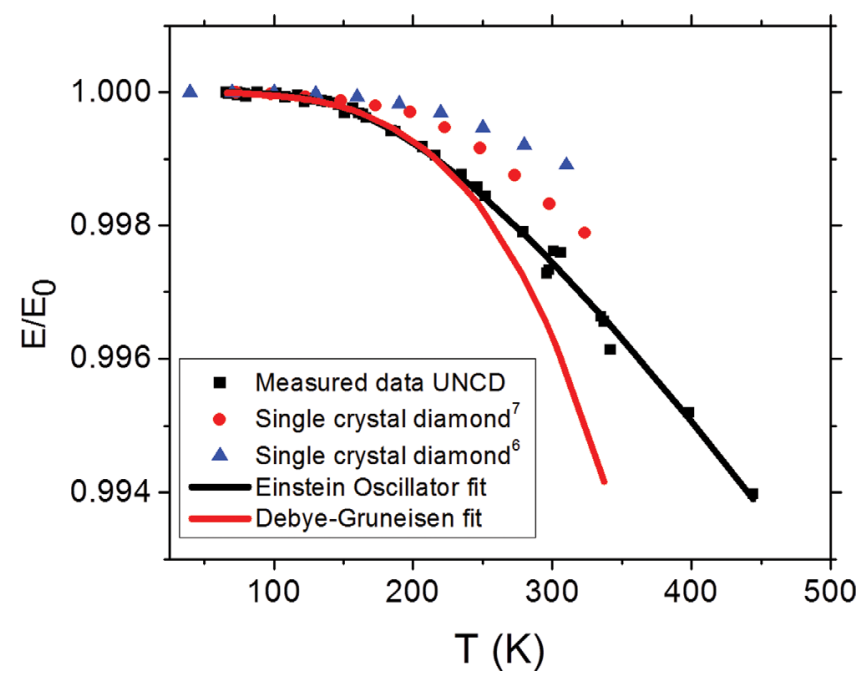

FIG. 3. (Color online) Temperature dependence of Young's modulus (relative to the low temperature limiting value) of UNCD (squares) and single crystal diamond (triangles ${ }^{6}$ and circles $^{7}$ ) averaged over all directions. Also shown are an Einstein oscillator fit and a Grüneisen-Debye fit for the UNCD data. The Grüneisen-Debye fit, derived for the low temperature limit, diverges at higher temperatures, as expected. bon atoms are $\mathrm{sp}^{3}$-bonded, and nanocrystalline diamond (NCD), ${ }^{16}$ which have larger grains (up to $300 \mathrm{~nm}$ ), have demonstrated a slightly higher reduction in Young's modulus (-33 ppm/K to $-50 \mathrm{ppm} / \mathrm{K}$ for $23^{\circ} \mathrm{C}<T<400{ }^{\circ} \mathrm{C}$ for NCD). Higher TCE in these materials is partly due to the higher base temperatures used for the measurement.

The elastic modulus of materials at $0 \mathrm{~K}$ directly depends on the energies and lengths of the atomic bonds. At finite temperatures, atoms absorb thermal energy and vibrate about their mean positions. The nearly harmonic potential within the bond lengths sampled at low temperatures leads to very little expansion in bond length or reduction in modulus, and hence the elastic modulus has nearly negligible temperature dependence. As the temperature increases, oscillations begin to sample a greater amount of the anharmonic character of the potential, which is asymmetrically wider at larger separations. This increases the amount of expansion of the bonds and the corresponding decrease in modulus. At higher temperatures comparable to the Debye temperature, most materials exhibit a linear decrease in Young's modulus with increasing temperature. Thus, a transition from an extended range of very little change in modulus to a linearly decreasing modulus occurs.

Wachtman et al. ${ }^{8}$ developed an empirical relation to describe the temperature dependence of Young's modulus, $E(T)$. Later, Anderson et al. ${ }^{9}$ derived a similar equation for the temperature-dependent bulk modulus $(B)$ by taking into account the anharmonic effects of lattice vibrations. We rewrite this equation in terms of the specific heat $c_{v}$ and $E(T)$ as,

$$
E(T)=E_{0}-\frac{3(1-2 \nu) \gamma \delta}{V_{0}} \int_{0}^{T} c_{v} d T
$$

where $E_{0}, V_{0}$ are the Young's modulus and the atomic volume at $0 \mathrm{~K}$, respectively; and $\gamma$ and $\delta$ are the Grüneisen parameter and the Anderson-Grüneisen parameter, respectively. This relation neglects temperature-dependent changes in $V_{0}$, the product $\gamma \delta$, and $\nu$. Thus, the derivative of $E$ with respect to $T$ strongly depends on $c_{v}$. At low temperatures, $c_{v}$ has a $T^{3}$ dependence and reduces to zero at $0 \mathrm{~K}$. Using the Grüneisen-Debye model for $c_{v}$, the resulting expression for $E$ is, ${ }^{17}$

$$
E(T)=E(0)-\frac{9(1-2 \nu) \pi^{4} R \Theta_{D} \gamma(3 \gamma-1)}{5 V_{0}}\left(\frac{T}{\Theta_{D}}\right)^{4}
$$

where $R$ is the ideal gas constant, and the Debye temperature $\Theta_{D}$ is given by: $\Theta_{D}=\frac{\hbar v_{D}}{k}\left(\frac{6 \pi^{2} N}{V_{0}}\right)^{\frac{1}{3}}$ where $N$ is Avogadro's number. It follows that the predicted rate of change of the elastic moduli with respect to $T$ decreases at low temperatures, and is zero at $0 \mathrm{~K}$. From our room temperature measurements of elastic modulus $(790 \mathrm{GPa})$ and Poisson's ratio $(0.057),{ }^{10}$ we obtain a Debye velocity $\left(v_{\mathrm{D}}\right)$ of $11254 \mathrm{~m} / \mathrm{s}$ and $\Theta_{D}$ of $1878 \mathrm{~K}$ (assuming $V_{0}=3.42 \mathrm{~cm}^{3} / \mathrm{mol}$, the value for single crystal diamond). Curve fitting our data (Fig. 3(b)) using Eq. (4) up to $T=\Theta_{D} / 10$ yields $\gamma=1.623$. As a result of the high value of $\Theta_{D}$, UNCD follows Eq. (4) very close to 
room temperature. In the high temperature limit, $c_{v}$ saturates (to $3 R$, where $R$ is the universal gas constant), and there is a linear decrease in modulus with increasing temperature. Using the Einstein oscillator expression for temperature dependence of $E{ }^{18}$

$$
E(T)=E_{0}-\frac{3(1-2 \nu) s}{\left(\exp \left(\frac{\Theta}{T}\right)-1\right)},
$$

where $\Theta$ is the effective Einstein temperature and $s$ is a parameter related to the zero point vibration energy contribution to elastic stiffness. Taking the derivative of $E$ in the high temperature limit yields, ${ }^{19}$

$$
\frac{d E}{d T}=-\frac{3(1-2 \nu) s}{\Theta}=\frac{9 R(1-2 \nu) \gamma(\gamma+1)}{V_{0}} .
$$

Curve fitting our data (Fig. 3(b)) to Eq. (4) yields $s=6.62$ $\mathrm{GPa}$, and $\Theta=682 \mathrm{~K}$. Solving Eq. (6) for $\gamma$ yields 0.76 . For single crystal diamond, $\gamma$ values estimated from Eqs. (4) and (6) were 1.26 and 0.71 , respectively, ${ }^{7}$ indicating that grain boundaries in UNCD increase the anharmonic parameter, as expected.

Developing a theoretical treatment of the temperaturedependent softening in UNCD requires understanding the contribution of grain boundaries to the vibrational density of states (VDOS), and hence $c_{v}$. Carbon atoms at the grain boundaries may be two-fold $\left(\mathrm{sp}^{1}\right)$ or three-fold $\left(\mathrm{sp}^{2}\right)$ coordinated in addition to being four-fold $\left(\mathrm{sp}^{3}\right)$ coordinated; these bonds may form at strained lengths or angles; and some bonds may be unterminated or terminated by hydrogen atoms. Similarly, the local energetics of $\mathrm{sp}^{3}$-bonded atoms close to the grain boundaries are influenced by the atoms at the grain boundaries. As a consequence, the local stiffness in regions of $\mathrm{sp}^{1}$ and $\mathrm{sp}^{2}$ bonding could be significantly lower than in regions of pure $\mathrm{sp}^{3}$ bonding. ${ }^{2}$ These bonds are more compliant than $\mathrm{sp}^{3}$ bonds, and accordingly, they exhibit higher anharmonicity. Thus, they would be expected to be the main contribution to the slightly higher temperature dependence of the UNCD modulus as compared to single crystal diamond. The temperature dependence of $c_{v}$ and $B$ of single crystal diamond and graphite have been theoretically calculated. ${ }^{19,20}$ Graphite (which is $\mathrm{sp}^{2}$-bonded) has a higher $c_{v}$ than diamond (which is $\mathrm{sp}^{3}$-bonded) at temperatures between $0-600 \mathrm{~K}^{21}$ The temperature dependence of vibrational properties of UNCD has been determined recently through molecular dynamic simulations, ${ }^{22}$ which indicate an increase of $\sim 20 \%$ in the specific heat at room temperature compared with single crystal diamond. ${ }^{22}$ The increase is predominantly due to the contributions from atoms at grain boundaries. Further efforts are needed to fully characterize the influence of atoms at grain boundaries on the temperature dependence of elastic properties of UNCD films.

The measured dissipation $\left(Q^{-1}\right)$ of the UNCD cantilevers varied from $(0.63-2.0) \times 10^{-4}$ with the relaxation of defects at the grain boundaries dominating the observed dissipation at room temperature. ${ }^{10}$ This is demonstrated by the fact that other dissipation mechanisms, including extrinsic mechanisms such as clamping losses, or intrinsic mechanisms such as thermoelastic dissipation, contribute negligibly to the observed dissipation, as discussed in detail previously. ${ }^{10}$ Clamping losses contribute negligibly to the observed dissipation based on predictions from models, and from the fact that experimentally measured $Q$ values of single crystal silicon $^{13}$ and polycrystalline diamond ${ }^{16}$ cantilevers with essentially the same clamping geometries as the cantilevers studied here were several orders of magnitude larger than those measured here. Our recent temperature-dependent measurements of $Q^{-1}$ showed a weak temperature dependence from $300 \mathrm{~K}$ down to $140 \mathrm{~K}^{23}$ To explore this further, dissipation was measured over a broader temperature range here, from $63 \mathrm{~K}$ to $450 \mathrm{~K}$ (Fig. 4). Consistent with previous measurements, the results show a moderate reduction in dissipation $Q^{-1}$ below $300 \mathrm{~K}$ and a very weak dependence below $180 \mathrm{~K}$. Previous temperature-dependent measurements shown in Fig. 4 of ta-C (Ref. 24) and NCD (Ref. 25) films deposited on silicon double paddle oscillators also showed a weak temperature dependence below $100 \mathrm{~K}$, followed by a weak power law dependence $\left(T^{\beta}, \beta<1\right)$ below $\sim 2 \mathrm{~K}$. Low temperature $(<10 \mathrm{~K})$ measurements of dissipation in metal-coated UNCD fixed-fixed beams $\left(f_{0}=5.1 \mathrm{MHz}\right)$ at temperatures below $5 \mathrm{~K}$ indicated a weak temperature dependence $(\beta=0.35,<5 \mathrm{~K})$ followed by a plateau above $5 \mathrm{~K} .{ }^{26}$ This behavior has been attributed to the presence of two level tunneling states ${ }^{26}$ and has been observed in many disordered materials, including glasses and amorphous forms of carbon, silicon, and silicon nitride, ${ }^{24-27}$ wherein stresses strongly determine the observed dissipation. $^{28}$ This disorder in UNCD mainly comes from defects at the grain boundaries. While we do not clearly resolve a plateau, the change in slope of $Q^{-1}$ versus

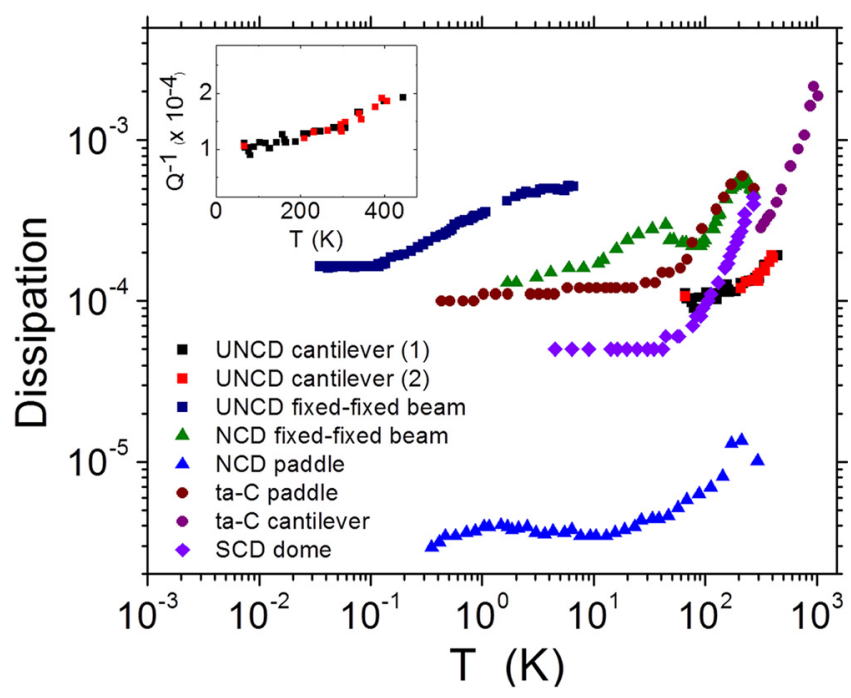

FIG. 4. (Color online) A comparison of dissipation as a function of temperature for carbon based resonators. Lever 1 (black squares) had no overhang (the resonant frequency shift of this cantilever is shown in Fig. 2), and Lever 2 (red squares) had an overhang of $\sim 30 \mu \mathrm{m}$ (the initial overhang was reduced to this value using FIB). Data include both heating and cooling measurements; no hysteresis is evident. Also plotted are data of UNCD fixed-fixed beams $\left(5 \mathrm{MHz}\right.$, flexural), ${ }^{26} \mathrm{NCD}$ paddle oscillators $(\sim 5.5 \mathrm{KHz}$, torsional), ${ }^{25} \mathrm{NCD}$ fixed-fixed beams $\left(13.7 \mathrm{MHz}\right.$, flexural), ${ }^{30}$ ta-C paddle oscillators $(\sim 5.5 \mathrm{KHz}$, torsional $),{ }^{24}$ cantilevers $(\sim 60 \mathrm{KHz} \text {, flexural })^{29}$ and single crystal diamond (SCD) dome resonators $\left(50 \mathrm{MHz}\right.$, flexural). ${ }^{32}$ Inset: UNCD cantilever data on a linear scale indicating the change in slope. 
temperature is consistent with a reduction in the rate of change in dissipation appearing at the lowest temperatures.

Overall, the UNCD measured here had dissipation values at low temperatures $\left(Q^{-1} \sim 10^{-4}\right)$ higher than the values observed for polycrystalline NCD torsional oscillators. ${ }^{25}$ However, the values were lower than those for amorphous ta-C (Ref. 24) torsional oscillators and flexural stress free cantilevers, ${ }^{29}$ indicating the contribution of defects. A comparison of dissipation in crystalline diamond and tetrahedral amorphous carbon resonator structures is shown in Fig. 4. Unlike our results in Fig. 4 for stress free monolithic UNCD cantilevers, dissipation in composite torsional paddles ${ }^{24,25}$ or high frequency $(>5 \mathrm{MHz})$ metal coated fixed-fixed beams $^{26,27}$ and membranes can be influenced by stress ${ }^{28}$ (due to differential thermal expansion), dissipation in metal coating, ${ }^{31}$ or clamping losses in membranes. ${ }^{32}$ Nonetheless, all of these results point to a much reduced temperature dependence of dissipation in these materials at low temperatures followed by a stronger dependence at very low temperatures $(<1 \mathrm{~K})$. The present results for UNCD are consistent with the idea that disorder at the grain boundaries are the dominant source of dissipation observed in UNCD. Tuning the grain boundary structure and film stress are therefore the most direct routes to controlling and potentially increasing the $Q$ of pure UNCD resonators at room temperature.

\section{CONCLUSIONS}

In summary, the Young's modulus and mechanical dissipation of UNCD films exhibit a weak dependence on temperature from $450 \mathrm{~K}$ to $63 \mathrm{~K}$. The temperature dependence of $E$ at low temperatures is within $10 \%$ that of single crystal diamond, significantly lower than that of amorphous carbon and many other materials of interest for MEMS/NEMS. This opens up new opportunities for using UNCD films in thermally stable resonators, sensors, and AFM probes. At low temperatures, the dissipation shows a weak temperature dependence similar to the observed behavior in tetrahedral amorphous carbon and nanocrystalline diamond films, indicating that dissipation in UNCD is dominated by defects within the film, i.e., at grain boundaries. Therefore, with respect to variations in temperature, UNCD has the interesting characteristic of behaving like a crystalline material in terms of modulus, but like a disordered material in terms of dissipation. Controlling the residual stresses and the grain boundary structure are key to achieving stable high frequency, high- $Q$ UNCD resonators for MEMS/NEMS, mass sensing, and other novel applications.

\section{ACKNOWLEDGMENTS}

This project was supported by DARPA under Grant No.06-W238, and was partially supported by the Nano/Bio Interface Center at the University of Pennsylvania through the National Science Foundation NSEC DMR08-32802. We thank G. E. Wabiszewski for proofreading the manuscript, and Kylan Szeto for help with curve fitting and for proofreading the manuscript.

${ }^{1}$ F. D. D. Tolla and K. W. Jacobsen, Nature 391, 561-563 (1998).

${ }^{2}$ I. N. Remediakis, G. Kopidakis, and P. C. Kelires, Acta Mater. 56, 5340 (2008).

${ }^{3}$ O. Auciello, S. Pacheco, A. V. Sumant, C. Gudeman, S. Sampath, A. Datta, R. W. Carpick, V. P. Adiga, P. Zurcher, Z. Ma, H. C. Yuan, J. A. Carlisle, B. Kabius, J. Hiller, and S. Srinivasan, IEEE Microw. Mag. 8, 61 (2007).

${ }^{4}$ W. Yang, O. Auciello, J. E. Butler, W. Cai, J. A. Carlisle, J. E. Gerbi, D. M. Gruen, T. Knickerbocker, T. L. Lasseter, J. N. Russell, L. M. Smith, and R. J. Hamers, Nat. Mater. 1, 253 (2002).

${ }^{5}$ J. A. Carlisle, Nat. Mater. 3, 668 (2004).

${ }^{6}$ H. J. McSkimin and P. Andreatch, J. Appl. Phys. 43 (7), 2944 (1972).

${ }^{7}$ A. Migliori, H. Ledbetter, R. G. Leisure, C. Pantea, and J. B. Betts, J. Appl. Phys. 104, 053512 (2008).

${ }^{8}$ J.B. Wachtman, W.E. Tefft, D. G. Lam, and C. S. Apstein, Phys. Rev. 122 (6), 1754 (1961).

${ }^{9}$ O. L. Anderson, Phys. Rev. 144 (2), 553 (1966).

${ }^{10}$ V. P. Adiga, A.V. Sumant, S. Suresh, C. Gudeman, O. Auciello, J. A. Carlisle, and R. W. Carpick, Phys. Rev. B 79, 245403 (2009).

${ }^{11}$ See supplementary material at http://dx.doi.org/10.1063/1.3693308 for temperature dependence of resonant frequency.

${ }^{12}$ D. M. Gruen, Annu. Rev. Mater. Sci. 29, 211 (1999).

${ }^{13}$ U. Gysin, S. Rast, P. Ruff, E. Meyer, D. Lee, P. Vettiger, and C. Gerber, Phys. Rev. B 69, 045403 (2004).

${ }^{14}$ Stanislav Stoupin and Yuri V. Shvyd'ko, Phys. Rev. Lett. 104, 2-5 (2010).

${ }^{15}$ D. A. Czaplewski, J. P. Sullivan, T. A. Friedmann, and J. R. Wendt, Appl. Phys. Lett. 87, 161915 (2005).

${ }^{16}$ N. Sepulveda, J. Lu, D. M. Aslam, and J. P. Sullivan, J. Microelectromech. Syst. 17, 473 (2008).

${ }^{17}$ L. Girifalco, Statistical Mechanics of Solids (Oxford, New York, 2000) p. 132.

${ }^{18}$ G. Leibfried and L. Ludwig, Solid State Physics (Academic, New York, 1961) Vol. 12, p. 275.

${ }^{19}$ H. Ledbetter, Phys. Status Solidi B 81, 181 (1994).

${ }^{20}$ N. Mounet and N. Marzari, Phys. Rev. B 71, 205214 (2005).

${ }^{21}$ T. Tohei, A. Kuwabara, F. Oba, and I. Tanaka, Phys. Rev. B 73, 064304 (2006).

${ }^{22}$ S. P Adiga, V. P Adiga, R. W. Carpick, D. W. Brenner, J. Phys. Chem. C 115 (44), 21691 (2011).

${ }^{23}$ V. P. Adiga, A. V. Sumant, S. Suresh, C. Gudeman, O. Auciello, J. A. Carlisle, and R. W. Carpick, Proc. SPIE 7318, 731818 (2009).

${ }^{24}$ X. Liu, T. Metcalf, P. Mosaner, and a. Miotello, Phys. Rev. B 71, 155419 (2005).

${ }^{25}$ T. H. Metcalf, X. Liu, B. H. Houston, J. W. Baldwin, J. E. Butler, and T. Feygelson, Appl. Phys. Lett. 86, 081910 (2005).

${ }^{26}$ M. Imboden and P. Mohanty, Phys. Rev. B 79, 125424 (2009).

${ }^{27}$ R. Pohl, X. Liu, and E. Thompson, Rev. Mod. Phys. 74, 991 (2002).

${ }^{28}$ D. R. Southworth, R. A. Barton, S. S. Verbridge, B. Ilic, A.D. Fefferman, H. G. Craighead, and J. M. Parpia, Phys. Rev. Lett. 102, 4 (2009).

${ }^{29}$ D. A. Czaplewski, J. P. Sullivan, T. A. Friedmann, and J. R. Wendt, Diamond Relat. Mater. 15, 309 (2006).

${ }^{30}$ A. B. Hutchinson, P. A. Truitt, K. C. Schwab, L. Sekaric, J. M. Parpia, H. G. Craighead, and J. E. Butler, Appl. Phys. Lett. 84, 972 (2004).

${ }^{31}$ C. Seoanez, F. Guinea, and A Castro Neto, Phys. Rev. B 77, 125107 (2008).

${ }^{32}$ M. P. Ray, T. I. Feygelson, J. E. Butler, J. W. Baldwin, B. H. Houston, B. B. Pate, and M. K. Zalalutdinov, Diamond Relat. Mater. 20, 1204 (2011). 